\title{
Perspectivas de las spin-off académicas, como modelo de emprendimiento en las universidades colombianas
}

\section{Academic spin-offs perspectives, as model for entrepreneurship in Colombian universities}

\author{
Saulo Bravo-García (iD \\ Universidad Santiago de Cali, Cali, Colombia \\ Jennifer Benavides-Bustos (iD) \\ Universidad Santiago de Cali, Cali, Colombia \\ María Alejandra Wagner-Martínez iD \\ Universidad Santiago de Cali, Cali, Colombia. \\ José Londoño-Cardozo (iD \\ Universidad Nacional de Colombia, Sede Palmira, Colombia.
}

\begin{abstract}
Resumen
\end{abstract}
Objetivo: proponer un esquema en el que se detalle los componentes ideales para la generación de una spin-off académica en Colombia. Método: se revisaron fuentes primarias y secundarias de información. Las primeras constaron de una consulta por medio de un cuestionario digital y telefónico que aportó información relacionada con cada una de las spin-off de las universidades que atendieron el llamado de la investigación, las segundas fueron, en esencia, documentos encontrados en las bases de datos académicas consultadas. Resultados: el esquema de spinoff propuesto a partir de la investigación puede ser replicado en todas las universidades del país. Para ello deben promoverse políticas internas de emprendimiento y favorecer un entorno que permita la investigación universitaria en las facultades de administración con miras hacia una transdisciplinariedad. Discusiones: aun cuando se plantea que las spin-off son modelos de desarrollo empresarial a partir de la investigación, se propone la búsqueda de generación de este tipo de organizaciones que permitan solventar las necesidades de la sociedad en el marco de la economía creativa y los modelos de economía colaborativa. Conclusiones: la spin-off como figura de generación de empresa es productiva y necesaria para el entorno del país y las políticas de fomento de Colciencias y de cada universidad sirven como semillero para este propósito. Además, el papel que la investigación juega es determinante dado que este tipo de organización deviene de los impulsos investigativos de las universidades en diferentes áreas del conocimiento.

Palabras clave: Spin off Académico, Modelo Spin Off; Emprendimiento en Colombia; Instituciones de Educación Superior; Spinouts.

Clasificación JEL: A22, I23, J01, J44, L26, M13, 032. \section{Correspondencia}

Autores de

sbravo@usc.edu.co benavidesjennifer3@gmail.com malejawm95@hotmail.com jodlondonoca@unal.edu.co

Recibido: 22-01-19

Aceptado: $30-04-19$

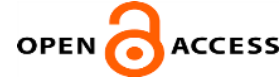

(cc) BY

Copyright (C) 2019

Desarrollo Gerencial

\begin{abstract}
Objective: this paper aims to propose a scheme in which ideal components to generate an academic spin-off in Colombia are detailed. Method: primary and secondary sources of information were reviewed, as follows: The first ones got through provided information in relation with the spin-off from those universities that applied for the call to research through a digital and telephone questionnaire; unlike, the second ones were, in essence, documents found based on academic data. Results: the proposed spin-off scheme from the research can be replicated with all universities of the country. According to this purpose, internal entrepreneurship policies and also, an appropriated environment in order to research in the faculties of administration taking into account transdisciplinary fields, should be promoted. Discussions: although spin offs are suggested as models of business development, the continous search for generating this type of organizations able to solve society needs based on a creative and collaborative economy models is proposed. Conclusions: spin-off as business generation becomes productive and necessary not only for the country's business environment, Colciencias promotion policies, but also to promote university incubators focus on this matter. In addition, this research plays a determinant role taking into account that this type of organization is the result from researches in different areas of knowledge conducted by universities. Keywords: Academic spin-off, Spin Off Model, Colombian Entrepreneur, Higher education Institutions, Spinouts. JEL Classification: A22, I23, J01, J44, L26, M13, 032.
\end{abstract}

Como citar este artículo (Apa):

Bravo-García, S., Benavides-Bustos, J., Wagner-Martínez, M. \& Londoño-Cardozo. J. (2019). Perspectivas de las spin-off académicas, como modelo de emprendimiento en las universidades colombianas. Desarrollo Gerencial, 11(1), 131-156. DOI: https://doi.org/10.17081/dege.11.1.3443 


\section{Introducción}

Las spin-off son un nuevo tipo de organizaciones creadas a partir del conocimiento generado desde la academia y la investigación (Méndez, Rebollar, Lino \& González, 2015), fungen desde las organizaciones bases, normalmente universidades, como mecanismo para la transmisión del conocimiento científico/tecnológico (Beraza \& Rodríguez, 2010). En el contexto colombiano, este tipo de organizaciones se encuentran en boca de todos los encargados de la generación de empresa dentro y fuera del ámbito académico, dada la promoción que en la actualidad entidades como Colciencias, o las universidades, hacen para este propósito. Es por esta razón que el objetivo de este documento, es presentar los resultados de una investigación, realizada en la Universidad Santiago de Cali, que tuvo como fin estudiar la generación de nuevas organizaciones, principalmente aquellas que tienen fines lucrativos, desde la academia bajo la figura de los spin-off.

Esta investigación se enfocó en establecer los diferentes componentes del modelo spin-off, además barca desde los fundamentos y antecedentes teóricos del modelo identificados a través de una revisión de literatura, hasta las políticas de fomento por parte de diferentes universidades colombianas. La investigación concluye con la propuesta de un esquema de spin-off ideal, posible de replicar en las universidades con sus respectivas adaptaciones.

De este modo, y gracias a la comprensión del desarrollo organizacional y su constante necesidad de actualización y generación de oportunidades, se especificarán las diferentes características, las bases y políticas establecidas en el país para la promoción de este tipo de organización. Así, el sentido final del estudio es presentar los beneficios que conlleva la formación de organizaciones bajo el modelo de spinoff. Estos son palpables tanto para los diferentes sectores económicos, como para los emprendedores. Asimismo, se destaca el impacto positivo de la aplicación adecuada de este modelo en ámbitos académicos, sociales y económicos.

De acuerdo con lo anterior, el documento se ordena de la siguiente manera: en primer lugar, se presenta un análisis previo sobre la literatura científica que permitió visualizar la aproximación conceptual a un modelo de spin-off académico. En este apartado se muestran, además, los diferentes tipos de organización subsumidos en esta categoría y sus principales características; en segundo lugar, se plantea la metodología empleada durante el estudio; en tercer lugar, se evidencian los principales resultados encontrados, su análisis y la discusión planteada; en seguida se planteará un modelo de spin-off resultado del estudio y finalmente se presentan las conclusiones pertinentes. 


\section{Fundamentación teórica}

En este apartado se presenta el sustento teórico empleado para la investigación. Primeramente, se aborda el concepto de spin-off desde diferentes autores, seguido se presentan los dos tipos posibles de este tipo de organización y finalmente la definición especifica de las spin-off académicas.

\section{Concepto de Spin Off.}

Las spin-off son iniciativas empresariales promovidas por miembros de la comunidad universitaria. En estas organizaciones se aplican los diferentes tipos de conocimiento que se desarrollan o se adquieren en el trasegar académico. Por lo anterior, existe cierta ambigüedad en el término (Méndez et al., 2015; Narváez, Maridueña, Chávez \& González, 2016) que será discutido a continuación.

En primer lugar, se debe señalar el origen semántico de la palabra, evidentemente spin-off es un término de origen anglosajón ampliamente utilizado en diferentes contextos. Su significado común, para todos los usos, es el de algo creado con base o a partir de algo ya existente (Méndez et al., 2015). En español, la traducción dada para este término es el de escindir. Su significado, a partir del diccionario de la Real Academia Española (2014), es el de cortar, dividir o separar algo para la creación de otra parte. Sin embargo, en los contextos académicos y empresariales se prefiere el uso del anglicismo. Por tal motivo, de forma precedente, se discuten algunas acepciones de uso académico.

Es menester aclarar que en este documento se emplea el concepto de organización en lugar del concepto de empresa dada la profundidad conceptual del primero. A partir de Tello (2018), se entiende a "la organización como un componente primordial para el funcionamiento del sistema social" (p. 100); de Dávila (2001) "como el ente social creado de forma intencional para el logro de determinados objetivos a través de la transformación de los recursos disponibles" (p. 6) y de Etzioni (1997) como las "unidades sociales (o agrupaciones humanas) deliberadamente construidas o reconstruidas para alcanzar fines específicos" (p. 4). Asimismo, Londoño y Hernández (2018) argumentaron que las empresas son un tipo de organización que tiene fines de lucro y como tal, toda empresa es una organización pero, no toda organización es una empresa. En líneas posteriores se observará que las spin-off no necesariamente se refieren a organizaciones con fines de lucro.

De esta manera, Nlemvo, Pirnay y Surlemont (2002) sostienen que una spin-off es una organización creada para explotar productos, servicios, procesos o desarrollos tecnológicos en centros de investigación, que por lo general, se conforma alrededor de una tecnología e individuos que formaban parte de la 
Saulo Bravo, Jennifer Benavides, María Wagner, José Londoño

organización matriz que dio origen al proceso, producto o tecnología (Golob, 2003). En su mayoría, estas se crean a partir de dos entes principales: a) Organizaciones Empresariales que realizan procesos de I+D y b) Organizaciones de Educación Superior (Golob, 2006).

Las primeras organizaciones de este tipo, y el uso del término en este contexto se presentaron en las década de los 70 del siglo XX. El desarrollo tecnológico en esta época en el Silicona Valley, tuvo como una de sus consecuencias la creación de organizaciones empresariales de base tecnológica a partir de las investigaciones de las universidades en California, Estados Unidos (Naranjo, 2011). Bajo este contexto, surgió lo que Tang, Vohora y Freeman (2004) definieron como organizaciones dependientes de los derechos de propiedad intelectual de las universidades. Asimismo, Iglesias, Jambrino y Peñafiel (2012) consideraron a las spin-off como un tipo de organización creada a través del conocimiento y la tecnología desarrollada desde la investigación en las universidades.

En este sentido, las spin-off sirven como plataforma para que el conocimiento fomentado a través de la investigación y el desarrollo tecnológico sea aplicado en un contexto real de la sociedad (Betancur \& Garay, 2015). Sin embargo, aunque la mayoría de los conceptos señalan a las universidades como las precursoras de las spin-off, otros autores conceptualizan a favor de su origen netamente empresarial. No obstante, Narváez et al. (2016) encontraron algunas similitudes que consideraron brindan cierta heterogeneidad dado que:

i) Se generan a partir de una organización existente; ii) se encuentran conformadas organizacionalmente por uno o varios individuos y iii) no necesariamente son empresas que utilizan la tecnología como motor para su funcionamiento. Bajo estas circunstancias parece conveniente precisar: cuando una empresa puede ser considerada de base tecnológica y cuando una spin off es universitaria, cuando es dependiente de una matriz y cuando no. (p. 97)

A partir de lo anterior, es necesario, identificar los diferentes tipos de spin-off que se pueden presentar en los contextos reales. Estos constituyen la base para la operación de una nueva actividad económica (Tübke, 2004).

\section{Tipos de Spin Off}

A pesar que el propósito de las spin-off es derivar el conocimiento generado en una organización A en una nueva organización $B$, existen situaciones en las que no se llega a generar una spin-off pues la organización resultante continua siendo parte de la organización inicial, aun cuando goza de cierta autonomía (Betancur, 2016). Por lo tanto, es importante tener en cuenta los factores que determinan la creación de los diferentes tipos de spin-off. 
Diferentes características y factores influyen en la creación y clasificación de las organizaciones (Londoño \& Hernández, 2018). Para las spin-off es posible destacar, entre otros factores: a) la actividad económica, b) el modelo de negocio, c) la gobernanza, d) los factores relacionales, e) factores de transferencia, f) los factores motivacionales y g) los factores ambientales (Tübke, 2004).

De la misma forma que no existe un consenso alrededor de la definición conceptual de spin-off, los tipos de organizaciones que pueden considerarse en esta categoría no resultan homogéneos (Druilhe \& Garnsey, 2004; Heirman \& Clarysse, 2004; Lowe, 2002; Mustar et al., 2006; Rodeiro, Calvo \& Fernández, 2012). El límite entre estos tipos de organización y otros suele ser confuso. Con respecto a lo anterior, Beraza y Rodríguez (2012) consideraron que "las spin-offs universitarias resultan ser fenómenos heterogéneos cuyas fronteras pueden variar de manera significativa según la percepción que sobre ellas tengan los agentes implicados sobre el terreno y los autores" (p. 41).

Entonces, realizar una taxonomía para las spin-off resulta problemático dada la flexibilidad de los límites (Beraza \& Rodríguez, 2012) y la marcada tendencia hacia la polisemia de términos para referirse a los diferentes tipos de organización. Este último, es un fenómeno recurrente para toda la disciplina administrativa (Ríos, 2010) y que a su vez, según Pirnay (2001), dificulta el análisis taxonómico. Sin embargo, a partir de la revisión realizada por Beraza y Rodríguez (2012) se puede proponer una taxonomía a partir de las características comunes de las organizaciones. La Tabla 1 muestra una síntesis de este resultado.

Tabla 1. Tipología de las spin-off

\begin{tabular}{llll}
\hline \multicolumn{1}{c}{ Categoría } & \multicolumn{1}{c}{ Tipos } & \multicolumn{1}{c}{ Autores proponentes } \\
\hline $\begin{array}{l}\text { Según la actitud de la } \\
\text { universidad con } \\
\text { respecto a las spin-off }\end{array}$ & $\begin{array}{l}\text { Spin-off espontáneas } \\
\text { Spin-off planificadas }\end{array}$ & OECD (2000), Pirnay (2001) y \\
& $\begin{array}{l}\text { Steffensen, Rogers y } \\
\text { (2000) }\end{array}$ & Speakman \\
\hline $\begin{array}{l}\text { Según el status de las } \\
\text { personas que han } \\
\text { dado origen a la idea }\end{array}$ & $\begin{array}{l}\text { Spin-off académicas } \\
\text { Spin-off de estudiantes }\end{array}$ & Bellini et al. (1999); Pirnay (2001); \\
\hline $\begin{array}{l}\text { Según si el } \\
\text { investigador se } \\
\begin{array}{l}\text { convierte en } \\
\text { emprendedor }\end{array}\end{array}$ & $\begin{array}{l}\text { Spin-off promovidas por el investigador } \\
\text { Spin-off promovidas por emprendedores } \\
\text { externos } \\
\text { pin-off ortodoxas } \\
\text { Spin-off híbridas }\end{array}$ & Smilor Gibson y Dietrich (1990) \\
\hline
\end{tabular}




\begin{tabular}{|c|c|c|}
\hline & \multicolumn{2}{|l|}{ Spin-off tecnológicas } \\
\hline & \multicolumn{2}{|l|}{ Spin-off dirigidas por el inventor } \\
\hline & \multicolumn{2}{|l|}{ Spin-off dirigidas por un comprador } \\
\hline & \multicolumn{2}{|l|}{ Spin-off dirigidas por un inversor } \\
\hline $\begin{array}{l}\text { Según si se transfiere } \\
\text { conocimiento } \\
\text { patentado }\end{array}$ & \multicolumn{2}{|l|}{$\begin{array}{l}\text { Spin-off basadas en tecnología patentada } \\
\text { Spin-off basadas en tecnología no patentada }\end{array}$} \\
\hline $\begin{array}{l}\text { Según la participación } \\
\text { de socios externos en } \\
\text { el capital }\end{array}$ & \multicolumn{2}{|l|}{$\begin{array}{l}\text { Spin-off con capital externo } \\
\text { Spin-off sin capital externo }\end{array}$} \\
\hline & \multicolumn{2}{|l|}{ Consultoría y contratos de I+D } \\
\hline $\begin{array}{l}\text { Según el tipo de } \\
\text { actividad }\end{array}$ & $\begin{array}{l}\text { Producto } \\
\text { Activos }\end{array}$ & $\begin{array}{l}\text { Druilhe y Garnsey (2004); Stankiewicz } \\
\text { (1994) }\end{array}$ \\
\hline & \multicolumn{2}{|l|}{ Licencia de la propiedad intelectual } \\
\hline $\begin{array}{l}\text { Según el modelo de } \\
\text { desarrollo seguido por } \\
\text { la spin-off }\end{array}$ & $\begin{array}{l}\text { Orientadas al crecimiento } \\
\text { No orientadas al crecimiento } \\
\text { Prospector spin-off }\end{array}$ & $\begin{array}{l}\text { Degroof (2002); European Commission } \\
\text { (2002) }\end{array}$ \\
\hline $\begin{array}{l}\text { Spin-off Corporativa o } \\
\text { Empresariales }\end{array}$ & $\begin{array}{l}\text { Las organizaciones que surgen desde iniciativas } \\
\text { empresariales }\end{array}$ & Tübke (2004) \\
\hline
\end{tabular}

Fuente: Elaboración propia (2019).

El origen es la característica en común que posee todas las categorías de spin-off antes enunciadas. Aunque clasificar una organización únicamente por la característica de su origen no resulta preciso (Londoño \& Hernández, 2018), en el caso que atañe a la investigación se puede utilizar para reconocer dos tipos de spin-off: 1) spin-off corporativas o empresariales y 2) spin-off académicas. A continuación, estas dos categorías se abordarán con mayor profundidad.

\section{Spin-Off Corporativas o Empresariales.}

Las Spin off corporativas corresponde a una nueva organización que surge a partir de una empresa matriz (Tübke, 2004). Esta figura se presenta en organizaciones que diversifican en sus modelos de negocio y emplean grandes cantidades de recursos para la investigación y el desarrollo (Montoya, 2016).

\section{Ejemplo:}

Mercadopago es una plataforma de pagos y transacciones online presente en Latinoamérica. Nació como el sistema de pagos de la plataforma de e-commerce MercadoLibre. Hoy en día es una empresa aparte constituida a partir de la experiencia desarrollada en MercadoLibre (El Tiempo, 2016). Ambas organizaciones son independientes y trabajan en alianza para concatenar sus servicios. 


\section{Spin-Off Académicas.}

Estas spin-off corresponden a las organizaciones que surgen desde la iniciativa académica y se dan a través de los procesos de investigación donde se manifiesta la transferencia del conocimiento científicotecnológico (Djokovic \& Souitaris, 2008; Montoya, 2016), es decir, la cultura de las spin-off desde el entorno universitario se proyecta como una alternativa para la construcción de una cultura empresarial (López, 2017) con la que se espera acelerar la generación de ideas de negocio desde los estudiantes emprendedores e innovadores (Pineda, Morales \& Ortíz, 2011; Rodeiro et al., 2012), con el fin de obtener un posicionamiento en el mercado. De esta manera, la creación de empresa desde la universidad bajo el modelo de spin-off se ha convertido en una tendencia con gran aceptación e interés que además es una estrategia para el cumplimiento de la misión institucional de las universidades (López, 2017; Naranjo, 2011) ya fomenta la cultura emprendedora a partir de centros tecnológicos y emprendedores con apoyo de la academia.

\section{Spin-off académica como sistema emprendimiento e innovación.}

Las universidades, además de la formación académica de estudiantes en diversas áreas y niveles de educación, intervienen a través de sus objetivos misionales en otros aspectos de la sociedad, como por ejemplo, las investigaciones para el desarrollo de nuevas pedagogías que contribuyen a la preparación de estudiantes en la resolución de problemas reales de organizaciones existentes durante su etapa de estudio (Bernal, 2019; Pinzón \& Pineda, 2017) y el fomento de la creación de los distintos tipos de organizaciones a través de las unidades de emprendimiento o incubadoras de empresas (Londoño, 2018).

Algunos autores consideran que no solo las investigaciones que conllevan una alta carga de innovación y tecnología de punta son susceptibles de convertirse en spin-off académicas (Degroof, 2002), sino que existen otros casos como Coolideres, ubicada en la ciudad de Tunja Colombia, la cual es una organización que funciona como una spin-off, no identificada como tal por sus socios, que adiestra a los estudiantes de administración de empresas en la resolución de problemas reales de una organización real (Bernal, 2019; Pinzón, 2016; 2019; Pinzón \& Pineda, 2017) que surgió desde las investigaciones de un modelo pedagógico para la disciplina (Bernal, 2019). En este mismo sentido, se resalta la importancia de las spinoff desde las incubadoras de empresas y para la dinamización de la economía a través del fomento empresarial.

Por otro lado, las spin-off suelen ir de la mano con el emprendimiento empresarial. Esto, principalmente por que el objeto del fomento de estas organizaciones desde la academia es que el 
Saulo Bravo, Jennifer Benavides, María Wagner, José Londoño

estudiante avance en el desarrollo de una iniciativa empresarial a través de la aplicación de los conocimientos adquiridos en el trasegar de su carrera. Todo ello se traduce en una actitud proclive para iniciar una empresa o, como lo afirmó Coase (1996), a convertirse en empresario. Con esto se busca, además de asegurar el futuro mismo de la persona, aportar al desarrollo económico del país mediante la soluciones a problemas sociales (Marulanda, Montoya, \& Vélez, 2014). Bajo este contexto se fundamenta la labor de las incubadoras de empresas quienes juegan un papel muy importante para la creación de las spin-off y la generación de empleos. Estas son organizaciones diseñadas para acelerar el crecimiento y asegurar el éxito de los proyectos emprendedores (Aneas, Sáez-Fuentes, \& Sáez-Rosenkranz, 2012). Su diseño organizacional está enfocado en un modelo que permite la generación de talentos con base en la investigación y la innovación. Su labor fundamental es el diseño de estrategias de investigación, desarrollo y comercialización de los productos generados en las empresas incubadas (Bravo, 2011, 2012).

\section{Método}

Para la realización del presente estudio se realizó la consulta de fuentes primarias y secundarias de información. Para el caso de las primeras se preparó un cuestionario dirigido a los representantes de las spin-off o delegados por parte de algunas de las universidades del país. Para las fuentes secundarias, se consultaron las bases de datos académicas con acceso desde la Universidad Santiago de Cali y la Universidad Nacional de Colombia, las páginas de internet institucionales de las universidades en estudio y las redes de difusión oficiales de las universidades. A demás, se utilizaron técnicas de análisis y síntesis documentales propias de las investigaciones cualitativas. El estudio se concentró en técnicas exploratorias de información, pero de manera general se implementaron técnicas descriptivas a partir de las encuestas y las entrevistas.

Asimismo, la búsqueda bibliográfica se centró en la identificación conceptual de las spin-off dentro del sistema de emprendimiento y organizacional, así como también su definición y su papel desde la investigación. Igualmente, permitió identificar la existencia de trabajos que abordaron de forma similar la temática del estudio. Los documentos encontrados durante la búsqueda en las bases de datos fueron analizados con la ayuda del software para investigaciones cualitativas Atlas. Ti, donde se agruparon por categorías de análisis los conceptos encontrados lo cual facilitó el desarrollo de la investigación.

El criterio para la selección de las universidades fue su pertenencia a la Red de Universidades de Emprendimiento. Esta decisión se sustentó a partir de la idea de que las universidades pertenecientes a esta red tienen una mayor propensión hacia la promoción de este tipo de modelos organizacionales. En total, ciento veinte universidades conforman la Red de Universidades de Emprendimiento, en adelante REUNE, las cuales se seleccionaron como muestra inicial para la investigación. Una vez se determinó 
Saulo Bravo, Jennifer Benavides, María Wagner, José Londoño

enfocar los esfuerzos en los miembros de esta red, se convocó una reunión con los miembros para realizar los contactos. Se debe aclarar que la Universidad Santiago de Cali, gestora de la investigación, hace parte de REUNE. El resultado de esta reunión permitió determinar catorce universidades de la base de datos de la red que contaban con spin-off académicas. El medio para contactar con estas universidades fue el correo electrónico. A través de este se les envío el cuestionario de la investigación. Este ejercicio arrojó como resultado la respuesta de dos universidades.

De las universidades que respondieron inicialmente el cuestionario, solo una manifestó contar con spin-off. Por este motivo fue necesario acceder a otras bases de datos universitarias. Por lo tanto la decisión final fue acceder a la información consolidada por Colciencias $(2017,2018)$ donde se relacionaban las páginas con proyectos de spin-off en Colombia.

En las universidades registradas en la base de datos de spin-off de Colciencias se registraron veinticinco participantes. Esta información es generada a partir de las políticas de promoción a la creación de nuevas empresas con base en la creatividad, la investigación y el de desarrollo de tecnologías (Colciencias, 2018). En esta base de datos se pudo observar la inclusión de las universidades reseñadas por REUNE lo que garantizó la equivalencia de ambas bases de datos. En esta etapa el proceso de contacto fue, en primer lugar, telefónico.

A partir de la información suministrada por Colciencias se procedió a contactar de forma directa a los encargados de las spin-off de cada universidad; finalmente se pudo establecer contacto con diecisiete universidades. A cada contacto se les solicitó un correo electrónico al cual se le envió el cuestionario digital para su diligenciamiento. En total diez universidades participaron en esta etapa.

\section{Estructura del cuestionario}

El cuestionario utilizado consto de veinticuatro preguntas divididas en cinco secciones que se reseñan en la Gráfica 1. Para el primer componente se agrupó dos preguntas. La primera acerca del conocimiento sobre la creación de empresas dentro del campus universitario con el apoyo institucional. La segunda, respecto a la misión. En esta pregunta se abordó la existencia de las spin-off como una alternativa de emprendimiento e innovación en la misión institucional de la universidad.

Para el caso del segundo componente se agruparon dos preguntas. La primera referente al reconocimiento de las spin-off como resultados de la investigación y la segunda relativa al tipo de investigación que las genera. 
El tercer componente subsumió diez preguntas. Estas giraron en torno al tipo de spin-off y se centraron en el funcionamiento interno y las características de las organizaciones resultantes. Se preguntó información relativa al periodo de existencia de la nueva organización, el tipo de sociedad conformada, el sector y el área a la que pertenece, los ingresos, la cantidad de empleados, entre otras cosas. Todo esto con la intención de caracterizar las spin-off universitarias generadas en el país.

En el cuarto componente se agruparon cuatro preguntas. Todas ellas giraron en torno al desarrollo y funcionamiento interno de la spin-off. Aquí, se indagó sobre la existencia de patentes, el apoyo presupuestal de la universidad hacia la organización naciente, entre otras. Finalmente, en el último componente se indagó sobre las fuentes de financiamiento de las spin-off y de la universidad para la promoción de estas organizaciones. Además, se consultó sobre la participación en redes de cooperación y articulación por parte de la spin-off.

En este componente también se preguntó sobre la importancia de factores como: capital humano, recurso financiero, recurso físico, recurso tecnológico, equipo de apoyo y apoyo institucional para el desarrollo de proyectos de spin-off. La importancia de algunos elementos como el apoyo del gobierno, la participación en redes o los sistemas de emprendimiento también fue objeto de consulta. Igualmente, se consultó sobre el porcentaje de recursos con los que cuenta la spin-off en cada una de las áreas funcionales de la organización, específicamente por la de mercadeo, producción, finanzas, recursos humanos, investigación y desarrollo.

\section{Gráfica 1. Secciones del cuestionario}

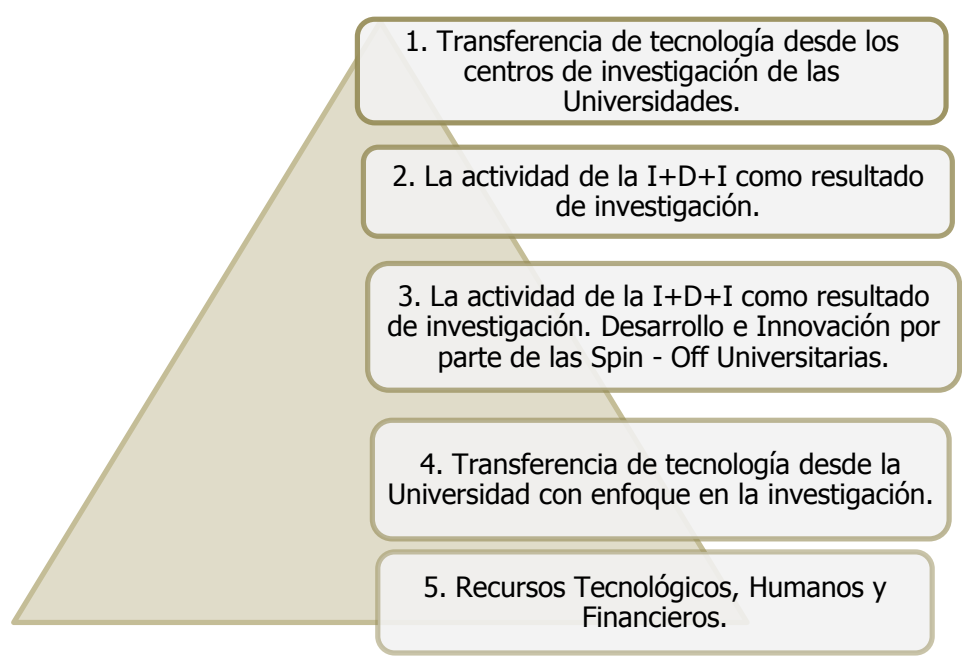

Fuente: Elaboración propia (2019). 
En el planteamiento inicial de la investigación se contempló el análisis de resultados a través de un modelo estadístico y el uso del Software SPSS. Sin embargo, en vista de los resultados, no fue posible la creación y el desarrollo de un modelo, pues la respuesta de las universidades no fue la esperada y la consistencia de los datos no era suficiente para llegar a resultados relevantes mediante ese procedimiento.

\section{Resultados y discusión}

Los resultados arrojados durante la investigación son bastantes significativos. Se pudo evidenciar que el apoyo de las universidades a la investigación y el fomento empresarial es determinante para promover esta modalidad de creación de empresa y de trabajo (Hernández, 2016). En este apartado primero se presentan y analizan los resultados del cuestionario realizado a las universidades seleccionadas. Posteriormente se hace una caracterización al modelo de las spin-off en Colombia para, finalmente, proponer un esquema o modelo ideal para las spin-off en las universidades del país.

En la primera pregunta se indagó acerca del mecanismo por el cual se enteraron que desde la universidad se podía crear empresa. En esta pregunta se pudo constatar que el $75 \%$ de los estudiantes conocieron que podían hacer empresa dentro del campus por medio de la promoción realizada por las universidades y a través de sus grupos de investigación. El 25\% restante tuvo contacto con las políticas de promoción empresarial en las universidades a través del contacto con otros estudiantes emprendedores, incluso de otros países y por medio de las revistas de innovación. Esto va en consonancia con las políticas de promoción del emprendimiento e innovación de las universidades miembros de REUNE. En este sentido el $100 \%$ de las respuestas a la segunda pregunta confirmaron la existencia de políticas de fomento empresarial en sus universidades y aceptaron que sus proyectos emprendedores fueron fruto de impulsos investigativos.

Se denota que la promoción de las políticas de emprendimiento al interior de las universidades tiene resultados loables. Aunque no fue posible identificar si en la totalidad de las universidades miembros de REUNE se hacía esta promoción, si se evidencia una relación directa entre cantidad de emprendimientos/ publicidad de las políticas respecto a las que no realizan divulgación alguna según la información consignada por la Red. Ahora bien, esto es objeto de otra investigación, indagar acerca de la promoción y la publicidad que las universidades realizan en temas de emprendimiento al interior de sus campus.

Con respecto al concepto de spin-off, todos los participantes aseguraron conocer que estas organizaciones son productos de la investigación, emprendimiento e innovación emanados de los 
Saulo Bravo, Jennifer Benavides, María Wagner, José Londoño

diferentes grupos que las conforman. Además, reconocieron la importancia del apoyo institucional dado por cada universidad para dicho propósito. Esto va acorde con las anteriores respuestas. Las universidades no solo publicitan o establecen políticas encaminadas al desarrollo del emprendimiento y la innovación dentro de la comunidad académica, sino que también se comprometen con el apoyo para la creación de las organizaciones resultantes.

En relación al sector económico, el 50\% de las spin-off que atendieron el llamado de la investigación se ubican en el sector agropecuario, el $25 \%$ sector industrial y el $25 \%$ servicios. El emprendimiento agropecuario y los diferentes avances emanados de la investigación se resaltan en este ítem. El interés por mejorar las condiciones y la competencia en el sector primario de la economía son plausibles y necesarios (Díaz \& Torrent, 2010).

En este sentido, la investigación desde las universidades colombianas parece atender al llamado de Zúñiga (2011) acerca de concentrar los esfuerzos en modernizar la labor en el campo y promover la creación de nuevos modelos de negocio agropecuarios y mejorar las condiciones de trabajo. Los datos entregados por los emprendedores evidencian que con sus spin-off contribuyen al sector agropecuario desde la generación y búsqueda de fuentes de energía amigables con el ambiente, en un 50\%, para biotecnología y nano tecnología y con un $25 \%$ de participación cada una.

El tipo de sociedad que se presenta en mayor medida en las spin-off que participaron en el estudio es la Sociedad Anónima Simplificada, SAS, con el 75\% de los casos; el 25\% restante está conformado por Sociedades anónimas. Esto es una señal que indica que, en mayor medida, estas organizaciones están constituidas por emprendedores, o nuevos empresarios, según León y Ríos (2017) y Zuluaga (2017). En igual medida, el 75\% de las spin-off constituidas por las universidades consultadas funcionan dentro de las mismas universidades. Los encargados de las spin-off manifestaron preferir continuar con su funcionamiento al interior de los campus universitarios dadas las garantías y la seguridad que esto significa. En general, la facilidad de acceso a nuevo conocimiento, laboratorios y mano de obra calificada, sin contar con los beneficios que la universidad pueda otorgar, le hacen preferir esta opción.

La ubicación de las spin-off es importante dado los altos costos en que se puede incurrir por este concepto. Algunas universidades tienen políticas de incentivo al emprendimiento cuando permiten, sea por un tiempo indefinido o determinado, que los impulsos emprendedores de sus estudiantes funcionen dentro del campus. Este sería un tema interesante para una futura investigación. Aun así, es posible mencionar que los encargados de las spin-off se declararon medianamente satisfechos con el apoyo de las universidades. Esto contrasta con un alto de satisfacción con el apoyo del sector público y muy poco satisfechos con el sector privado. 
En esencia, los emprendedores recurrieron a recursos de la universidad, recursos propios y a los diferentes programas de apoyo empresarial existentes. Este es el caso del Fondo emprender, INnpulsa, Ventures Capital, Waira, Destapa tu futuro y Colciencias. A partir de los datos se evidenció que solo Colciencias contribuyó en el financiamiento de los proyectos en un rango entre $51 \%$ a $80 \%$ y la universidad realizo una contribución entre un $21 \%$ a $50 \%$. A continuación, es necesario abordar el tema de la transferencia de conocimiento desde las spin-off hacia el sector en el que llevan a cabo su función social.

El estado de formalización de estos emprendimientos es aún prematuro. El rango de edad de todas las spin-off consultadas se encuentra entre uno y cinco años. Los ingresos mensuales son inferiores a cinco millones de pesos colombianos en el $75 \%$ de los casos y de once a cincuenta millones en el $25 \%$ restante. En general el promedio de puestos de trabajo generado por las spin-off se encuentra entre cinco y diez empleados, esta cifra varía debido al tiempo de existencia de cada organización.

Con relación a la transferencia de conocimiento, el $50 \%$ de las organizaciones consultadas manifestaron transferir conocimiento desde la organización hacia el sector de pertenencia. No obstante, asumir que la mitad de las spin-off no transfiere conocimiento al sector sería hilar muy delgado. Algunas de estas organizaciones se encuentran en sectores como la Responsabilidad Social, la biotecnología o en servicios de asesoría, donde no es tan evidente este proceso. Aun así, el 100\% de quienes manifestaron realizar transferencia de conocimiento lo hacen a través de ofrecer productos y servicios diferenciados. Aunque dos de las organizaciones manifestaron estar en procesos de solicitud de patentes, ninguna notificó la posesión de uno de estos títulos.

Modelo de las spin-off académicas como generador de transferencia de tecnología, innovación y emprendimiento

Para caracterizar el modelo general de spin-off académico en Colombia se propuso una matriz que rindiera cuenta del nivel de cumplimiento de las características propuestas para un spin-off en las cuatro organizaciones que aportaron toda la información. La Tabla 2 presenta el comparativo entre las organizaciones y su factor de cumplimiento.

En esta matriz se califica el cumplimiento de las características del modelo de spin-off para cada una de las organizaciones relacionadas. Para ello, cada característica fue calificada con una ponderación entre 1 a 5 puntos. La escala de cada calificación fue: a) 5 puntos para Cumplimiento, b) 4 puntos para Cumplimiento Significativo, c) 3 puntos para Cumplimiento Parcial, d) 2 puntos para Cumplimiento mínimo 
y e) 1 punto para No Cumplimiento. La organización con el mayor puntaje en la sumatoria total es la que más se aproxima en la práctica al modelo ideal de spin-off.

Tabla 1. Cumplimiento Modelo Spin Off Académicas

\begin{tabular}{ccccc}
\hline & $\begin{array}{c}\text { Spin-off } \\
\text { académicas } \\
\text { estudiadas }\end{array}$ \\
$\begin{array}{c}\text { Modelo } \\
\text { spin-off }\end{array}$ & AGRANOVA & $\begin{array}{c}\text { CARBÓN } \\
\text { ACTIVADO } \\
\text { ESPECIALIZADO }\end{array}$ & CONOSER & AMI-TEC \\
\hline Emprendedor & 5 & 5 & 5 & 5 \\
\hline Innovación & 5 & 5 & 5 & 5 \\
\hline Gestión desde la academia & 5 & 5 & 5 & 5 \\
\hline Transferencia de tecnología & 5 & 4 & 4 & 5 \\
\hline Participación en el mercado & 5 & 5 & 4 & 4 \\
\hline TOTAL & 25 & 24 & 23 & 24 \\
\hline
\end{tabular}

Fuente: Elaboración propia (2019).

Con base en el comparativo anterior se demuestra la participación de la academia en el fomento del desarrollo empresarial, la transferencia tecnológica, innovadora y participación en el mercado bajo el modelo de spin-off académica. Esto sustenta la intención del presente trabajo y de la investigación.

De este modo, es imperativo resaltar el trabajo realizado por la Universidad del Valle como alma mater de AGRANOVA y CARBON ACTIVO ESPECIALIZADO. Estas dos organizaciones poseen los resultados más altos en la escala de valoración que se realizó, con 25 y 24 puntos respectivamente. De igual manera, es de anotar que AGRANOVA se caracteriza por ser la spin-off académica más activa, lo que demuestra que este modelo de generación de empresa arroja grandes resultados y genera beneficios para el desarrollo empresarial y social del país. Esto a su vez, es sustentado por los soportes suministrados por AGRANOVA en los que se observa la gran actividad funcional y económica de la organización. Esta cuenta con diferentes alianzas estratégicas, específicamente con el grupo de Investigación ILAMA de la Universidad del Valle y el laboratorio de Física ambiental, cuyos desarrollos tecnológicos se encuentran avalados por Colciencias y patentados en Colombia. AGRANOVA posee un gran potencial en el mercado y ha sido merecedora de diversos premios y reconocimientos a nivel nacional en emprendimiento y como modelo spin-off académico exitoso en el país.

\section{Aproximación a un modelo spin-off}

A partir de los hallazgos encontrados y con base en la recopilación teórica realizada, se determinó proponer un esquema en el que se detalle los componentes ideales para la generación de una spin-off académica en Colombia. En la Gráfica 2 se establece el modelo ideal de una spin-off académica. 
Según Betancur (2016), las universidades justifican la promoción y el fomento de las spin-off como mecanismo de transferencia al sector productivo y social en beneficio del incremento de los índices de innovación y competitividad del tejido empresarial del país. Para esto, se establece en el modelo spin-off académica dos campos importantes; el entorno y la universidad. Estos comprenden diferentes aspectos relevantes, en donde se encuentra un marco legal que entiende las políticas que apoyan a las diferentes iniciativas de innovación y emprendimiento en Colombia.

Las Universidades están conformadas por diferentes facultades y programas académicos, dentro de los cuales se desarrollan grupos de investigación, semilleros o centros de emprendimiento. (Betancur \& Garay, 2015). Indudablemente la investigación es un factor trascendental para el desarrollo de spin-off desde las universidades. En este contexto es necesario revisar la conformación curricular de los programas de administración y de las facultades a las que estos programas están adscritos.

El equilibrio entre práctica y teoría en las facultades de administración y para el emprendimiento del que abordó Londoño (2018) se debe complementar con una buena y suficiente formación en investigación. El trabajo de Giraldo, Tello, Londoño, \& Pineda (2019) aporta argumentos en este sentido. Sin embargo, se debe mencionar que, aunque se propone examinar con profundidad los currículos de los programas de administración, se considera que el emprendimiento es transversal a varias disciplinas. Por lo tanto, es necesario revisar las alianzas transdisciplinares entre programas académicos y sus estrategias de investigación que permitan su aprovechamiento como spin-off al igual que AGRANOVA.

Las spin-off académicas pueden ser fundadas por los estudiantes, docentes, investigadores, funcionarios o por personas externas a la universidad que se dedican a transformar los conocimientos desarrollados dentro de estas en productos y servicios innovadores (Betancur \& Garay, 2015). Esto, da como resultado la transferencia tecnológica, de conocimiento, emprendimiento e innovación y genera la base para la operación de una nueva actividad económica (Tübke, 2004). 
Gráfica. 2 Propuesta de un modelo de spin-off para el contexto colombiano.
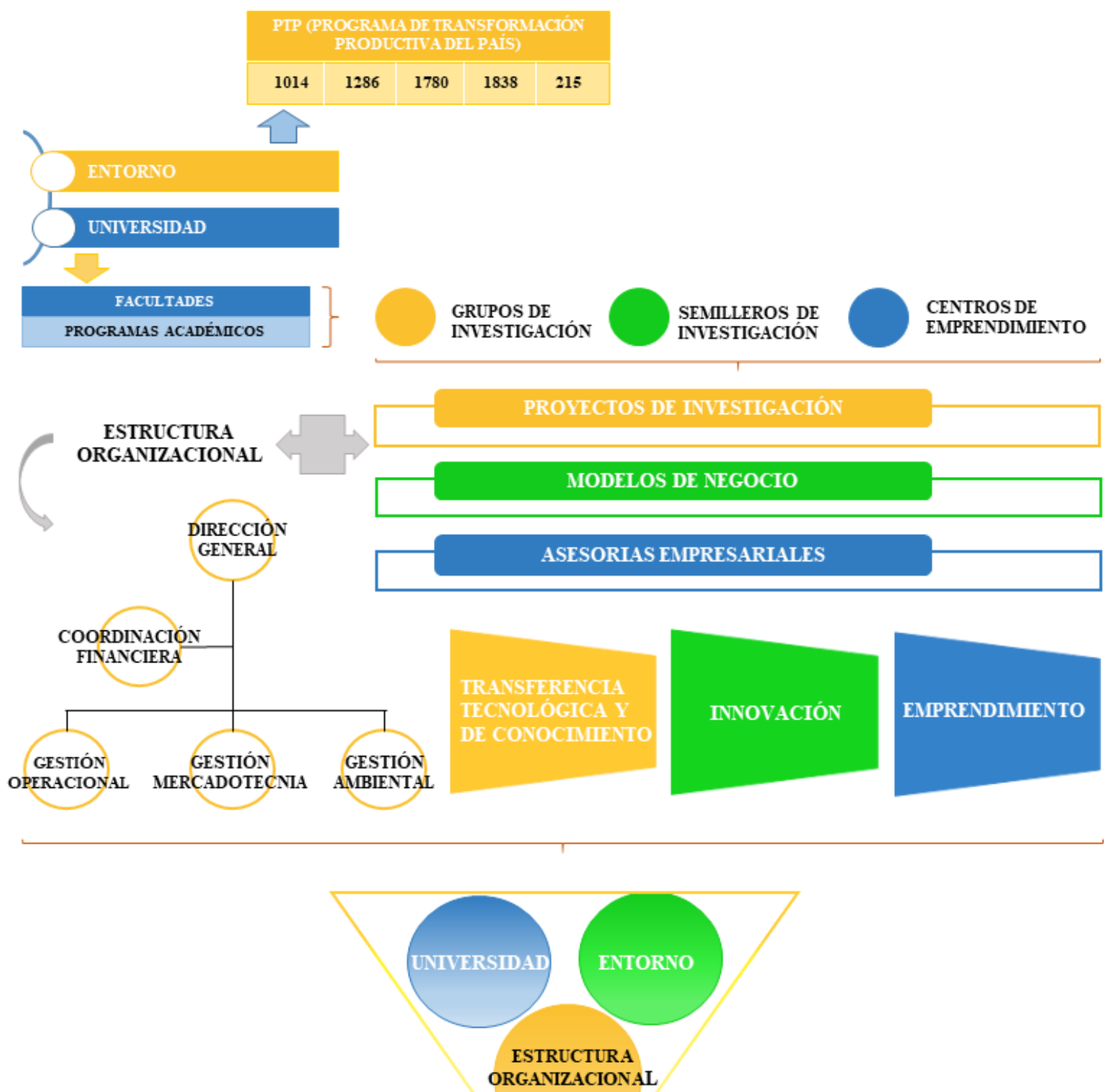

\section{SPIN-OFF ACADÉMICA}

Fuente: Elaboración propia (2019). 
La estructura de la organización debe ir acorde a la estrategia (Chandler, 1962). Por otra parte, se propone una estructura organizacional óptima y activa, que ahorre espacios, tiempos y movimientos en las actividades de cada miembro de la organización. En general, el tamaño de las spin-off gestadas es pequeño en sus primeros años. Por tanto, se recomienda una planificación acorde con esta característica.

La spin-off académica se presenta como la unificación del entorno, la universidad y la estructura organizacional. La implementación planteada origina una spin-off académica ideal, esto no desconoce que la premisa básica de la planeación estratégica y de la estructura es no generalizar o proponer supuestos ideales universales sino pautas que con sus respectivas adaptaciones a cada situación y entorno podría ser implementado. Por tanto, a partir de la investigación se sugiere: la generación de alternativas innovadoras a partir de las actividades academias y spin-off coherentes con el impulso bridado mediante las estrategias estatales regidas por las leyes de desarrollo empresarial que implican la cultura emprendedora (Ley 1014 de 2006), la transferencia tecnología o Ciencia tecnología e Innovación (Ley 1286 de 2009), la motivación de estímulos para que la vinculación de jóvenes investigadores a procesos que garanticen su independencia (Ley 1780 de 2016).

Un modelo de spin-off coherente con estas sugerencias facilita la posibilidad de trabajar con equipos interdisciplinarios mediante los grupos y semilleros de investigación desde donde se pueden brindar soluciones a problemas y considerar las necesidades de los objetivos de desarrollo sostenible que pueden dar surgimiento a las spin-off. En definitiva, al iniciar una startup el ente educativo debe: diseñar y aplicar la política de funcionamiento de este modelo de emprendimiento a partir del análisis de la política estatal e identificar oportunidades y focos de desarrollo sostenibles regionales, esto implicaría entonces la valoración del entorno lo que convierte a las universidades en un impulsor de estas unidades de desarrollo de innovación.

De esta manera, las universidades deben establecer currículos que permitan generar proyectos interdisciplinarios que involucren las competencias, las capacidades y recursos de las instituciones de forma integral al desarrollo de equipos interdisciplinarios de investigación conformados por profesores y estudiantes que, desde el aula de clase, puedan identificar problemas y los conviertan en insumos para la puesta en marcha de proyectos que apunten a tipos de Innovación Incremental, Radical y Disruptivos. Ello aportará a una transferencia de conocimiento con impulso de innovación transformacional en el sistema productivo.

Por otro lado, el fortalecimiento de estas unidades de innovación que se gestan desde las universidades requiere crecer con el tiempo. Adicionalmente, estas unidades requieren de las capacidades de las universidades por intermedio de los centros de desarrollo emprendimiento y consultoría 
Saulo Bravo, Jennifer Benavides, María Wagner, José Londoño

empresarial. En este contexto, el modelo de spin-off universitario necesita el desarrollo de un sistema de esta categoría.

El funcionamiento del sistema de unidades de emprendimiento e innovación demanda estructuras organizacionales coherentes con el desarrollo de Gestión del conocimiento. De la misma forma, las spinoff deberán estructurarse con equipos transdisciplinarios según sus necesidades de operación empresarial. Estos equipos deberán ser orientados a la gestión de actividades para la innovación sin perder la naturaleza académica que da respuesta a una necesidad del entorno. No obstante, se deberán incluir, unidades organizacionales como la administrativa, la financiera, de mercadotecnia, ambiental y de gestión de la Responsabilidad Social Organizacional.

\section{Conclusiones}

Un sistema académico universitario desde donde se desarrollen proyectos originados a partir del currículo y articulados con las misiones académicas, de acuerdo con las leyes de educación y desarrollo empresarial, orientadas a las necesidades del entorno, serian verdaderas cunas de emprendimientos innovadores bajo el modelo spin-off académicas. El emprendimiento como impulsor de la economía y generador de empleo es una buena práctica que debe promoverse desde las universidades. Son estas organizaciones las encargadas de generar el conocimiento necesario para incursionar en nuevos modelos y con nuevas tecnologías que contribuyan a la solución de las necesidades de las personas desde diferentes sectores económicos y sociales. Las spin-off que resultan de la integración, investigación y fomento empresarial llevado a cabo en las universidades es un claro ejemplo de ello.

Este modelo organizacional es fructífero para el avance socioeconómico regional con el objetivo principal de la satisfacción constante de las necesidades del consumidor de productos, servicios y soluciones sociales. Además, aportan puestos de trabajo calificados y promueven el empleo entre los universitarios.

Las spin-off poseen características fundamentales para su adecuado desarrollo y están sumadas a los aportes que las universidades realizan en este propósito. El fomento empresarial parece ser una constante en algunas de las universidades del país.

Por consiguiente, el modelo propuesto puede ser replicado en las universidades, aunque estas deben tener en cuenta algunas sugerencias. Es necesario promover, desde las universidades y desde el gobierno, las redes de investigación y académicas entorno a las spin-off. Los datos arrojaron que el 50\% 
Saulo Bravo, Jennifer Benavides, María Wagner, José Londoño

de las universidades participantes pertenecen a redes de investigación en el tema con lo que es necesario una mayor participación.

El principal aporte que dan las universidades para este propósito es el apoyo en temas como: el acondicionamiento de espacios para el surgimiento de la empresa, la ayuda en la identificación de oportunidades, la investigación de mercados y análisis de competidores, el desarrollo de planes de negocios, la formación del equipo y la trasferencia de tecnología. En este sentido es evidente que para la generación de spin-off académicas las universidades deben crear y consolidar una plataforma de investigación, emprendimiento e innovación.

Adicionalmente, se propone la revisión de las políticas de promoción al emprendimiento en las diferentes universidades. Esta modalidad de empleo puede ayudar a encontrar soluciones a los problemas sociales. Estas políticas no deben ir únicamente encaminadas hacia la generación de emprendimientos del tipo empresarial como los expuestos en este documento, sino que deben promover cualquier tipo de solución a los problemas sociales. Este es el caso de los emprendimientos en modelos de la Economía colaborativa que estudiaron Ospina, Cifuentes, Gaitán, y Londoño (2019) y Acosta (2017). En este orden ideas, sería importante analizar la factibilidad de la generación de spin-off dedicadas a los asuntos de Economía Creativa, mal llamada economía naranja (Hernández, Ospina, Londoño, \& Tello, 2019); economía colaborativa y emprendimientos sociales y no únicamente a los emprendimientos empresariales con fines de lucro aquí descritos.

De acuerdo con lo anterior, se proponen para futuras investigaciones, profundizar en el tema de las spin-off académicas. Especialmente lo referido a los tipos de investigación pertinentes para ello, a la profundización de las características del sistema institucional que soporta este modelo y del beneficio que perciben las universidades a partir de ello. Muchos otros temas podrán resultar de este proceso que será necesario abordar para consolidar este modelo. Evidentemente este modelo es muy general y será el objeto de futuras investigaciones ahondar en las especificidades del mismo.

\section{Referencias}

Acosta B. (Octubre, 2017). Consumo colaborativo: Una reflexión sobre su introducción desde el Neoinstitucionalismo. Trabajo presentado en el Congreso Reflexiones Sobre Administración. Tendencias actuales de investigación Administrativa, Palmira, Colombia. Recuperado de http://bdigital.unal.edu.co/70078/ 
Aneas, A., Sáez-Fuentes, E. \& Sáez-Rosenkranz, I. (2012). Emprendimiento en estudiantes de educación superior en chile: intervención pedagógica para fortalecer la innovación y la creatividad. En Pujol, M., Lorenzo, N. \& Violant, V. Innovación y creatividad: Adversidad y Escuelas creativas (p. 11). Barcelona: Universitat de Barcelona.

Bellini, E., Capaldo, G., Edström, A., Kaulio, M., Raffa, M., Ricciardi, M. \& Zollo, G. (1999). Strategic Paths of Academic Spin-Offs: A Comparative Analysis of Italian and Swedish Cases. Trabajo presentado en 44th ICSB Conference, Naples, USA.

Beraza, J. \& Rodríguez, A. (2012). Tipología de las spin-offs en un contexto universitario: una propuesta de clasificación. Cuadernos de Gestión, 12(1), 39-57. DOI: https://doi.org/10.5295/cdg.090181jb

Beraza-Garmendia, J. \& Rodríguez-Castellanos, A. (2010). Factores determinantes de la utilización de las spinoffs como mecanismo de transferencia de conocimiento en las universidades. Investigaciones Europeas de Dirección y Economía de la Empresa, 16(2), 115-135. DOI: https://doi.org/10.1016/S1135-2523(12)60115-4

Bernal-Campos, H. (2019). Didáctica para administración de empresas con laboratorio de empresa. En Beltrán-Duque, A. Echeverry-Romero, R. Restrepo-Rivillas, C. \& Rodríguez-Orjuela, R. Las nuevas tecnologías y los desafíos para la administración. $1^{\text {a }}$ Ed. (pp. 3180-3200). Bogotá: Fundación Universidad Externado de Colombia.

Betancur-Monsalve, M. \& Garay, K. (2015). En la senda de una hoja de ruta de Spin-Off universitarias en Colombia. Recuperado de http://www.spinoffcolombia.org/wp-content/uploads/2016/09/EN-LASENDA-DE-UNA-HOJA-DE-RUTA-DE-SPIN-OFF.pdf

Betancur-Monsalve, M. (2016). Hacia una Hoja de Ruta Spin-Off Colombia. Un camino para la creación de spin-off universitaria en Colombia. Recuperado de http://www.spinoffcolombia.org/hoja-de-ruta/

Bravo-García, S. (2012). Incubadoras de empresas, creación de empresas y redes sociales (Tesis doctoral en Administración y Economía, Universidad de Sevilla). Recuperado de https://idus.us.es/xmlui/bitstream/handle/11441/14890/M_TD-PROV15.pdf?sequence $=1$

Bravo-García, S. (2011). Incubadoras de empresas en Colombia. Revista Ingenium, 5(9), 5-13.

Chandler, A. (1962). Strategy and Structure: Chapters in the History of the American Industrial Enterprise (reprinted). United States of America: Beard Books. 
Coase, R. (1996). La naturaleza de la empresa. En Williamson, O. \& Winter, S. La Naturaleza de la Empresa: Orígenes, Evolución y Desarrollo (pp. 9-26). México: Fondo de Cultura Económica.

Colciencias. (2017). Conoce siete casos de éxito de proyectos SpinOff. Recuperado de https://www.colciencias.gov.co/sala_de_prensa/galerias/conoce-siete-casos-exito-proyectosspinoff

Colciencias. (2018). Competitividad para el desarrollo. Recuperado de SpinOff: http://www.spinoffcolombia.org/

Congreso de Colombia. (02 de mayo de 2016). Por medio de la cual se promueve el empleo y el emprendimiento juvenil, se generan medidas para superar barreras de acceso al mercado de trabajo y se dictan otras disposiciones. [Ley 1780 de 2016]. DO: 49.861.

Congreso de Colombia. (23 de Enero de 2009). Por la cual se modifica la Ley 29 de 1990, se transforma a Colciencias en Departamento Administrativo, se fortalece el Sistema Nacional de Ciencia, Tecnología e Innovación en Colombia y se dictan otras disposiciones. [Ley 1286 de 2009]. DO: 47241.

Congreso de Colombia. ( 26 de enero de 2006). Ley de fomento a la cultura del emprendimiento. [Ley 1014 de 2006]. DO: 46164.

Dávila, C. (2001). Teorías organizacionales y administración. Enfoque crítico. $2^{\text {da }}$ ed. Bogotá: Editorial Presencia LTDA.

Degroof, J. (2002). Spinning off new ventures from research institutions outside high tech entrepreneurial areas (Doctoral dissertation in Management, Massachusetts Institute of Technology). Recuperado de https://dspace.mit.edu/handle/1721.1/8477

Díaz-Chao, Á., \& Torrent-Sellens, J. (2010). ¿Pueden el uso de las TIC y los activos intangibles mejorar la competitividad? Un análisis empírico para la empresa catalana. Estudios de economía aplicada, 28(2), 1-22. Recuperado de http://www.redalyc.org/articulo.oa

Djokovic, D. \& Souitaris, V. (2008). Spinouts from academic institutions: A literature review with suggestions for further research. Journal of Technology Transfer, 33(3), 225-247. DOI: https://doi.org/10.1007/s10961-006-9000-4

Druilhe, C. \& Garnsey, E. (2004). Do Academic Spin-Outs Differ and Does it Matter? Journal of Technology Transfer, 24(3-4), 269-285. DOI: https://doi.org/10.1023/B:JOTT.0000034123.26133.97 
Etzioni, A. (1997). Organizaciones modernas [Traducción al Español] (1a ed.). México: Limusa NORIEGA EDITORES.

European Commission. (2002). University spin-outs in Europe. Overview and good practice. Luxemburgo: EU publications.

Giraldo-López, A. R., Tello-Castrillón, C., Londoño-Cardozo, J. \& Pineda-Henao, E. (2019). Influencia de la malla curricular en la formación investigativa en programas de administración en Colombia. Revista Argentina de Investigación en Negocios, 5(1), 19-32. Recuperado de http://ppct.caicyt.gov.ar/index.php/rain/issue/view/v5n1/showToc

Golob, E. (2003). Generating spin-offs from university-based research: an institutional and entrepreneurial analysis (Doctoral dissertation in Urban Planning). Rutgers University, New Jersey.

Golob, E. (2006). Capturing the Regional EconomicBenefits of University TechnologyTransfer: A Case Study. Journal of Technology Transfer, 31(6), 685-695. Recuperado de https://link.springer.com/article/10.1007/s10961-006-0023-7

Grandi, A., \& Grimaldi, R. (2005). Academics' organizational characteristics and the generation of successful business ideas. Journal of Business Venturing, 20(6), 821-845. DOI: https://doi.org/10.1016/j.jbusvent.2004.07.002

Heirman, A., \& Clarysse, B. (2004). How and Why do Research-Based Start-Ups Differ at Founding? A Resource-Based Configurational Perspective. Journal of Technology Transfer, 29(3-4), 247-268. DOI: https://doi.org/10.1023/B:JOTT.0000034122.88495.0d

Hernández-Madroñero, I., Ospina-Díaz, L., Londoño-Cardozo, J., \& Tello-Castrillón, C. (2019). Economía naranja o economía creativa. Una discusión conceptual respecto a la ley 1834 de 2017. En Beltrán- Duque, A., Echeverry-Romero, R., Restrepo-Rivillas, C. \& Rodríguez-Orejuela, A. Las nuevas tecnologías y los desafíos para la administración (pp. 427-449). Bogotá, Colombia: Fundación Universidad Externado de Colombia.

Hernández-Madroñero, I. (2016). Modalidades de trabajo. Working Paper, Palmira, Valle del Cauca.

Iglesias-Sánchez, P., Jambrino-Maldonado, C. \& Peñafiel-Velasco, A. (2012). Caracterización de las SpinOff universitarias como mecanismo de transferencia de tecnología a través de un análisis clúster. Revista Europea de Dirección y Economía de la Empresa, 21(3), 240-254. Recuperado de https://doi.org/10.1016/j.redee.2012.05.004 
León-Hernández, G. \& Ríos-González, S. (2017). SAS (sociedad por Acciones Simplificadas) como una alternativa para las micro, pequeña y mediana empresa: El emprendurismo. Horizontes de la Contaduría en las Ciencias Sociales, 4(7), 50-57. Recuperado de https://www.uv.mx/icp/revistahorizontes-numero-7/

Lockett, A. \& Wright, M. (2005). Resources, capabilities, risk capital and the creation of university spin-out companies. Research Policy, 34(7), 1043-1057. DOI: https://doi.org/10.1016/j.respol.2005.05.006

Londoño-Cardozo, J. \& Hernández-Madroñero, I. (2018). El problema de los conceptos científicos en la clasificación de las organizaciones. En Tello-Castrillón, C. \& Pineda-Henao, E. (Comp.), Conjeturas organizacionales. Fundamentos para el estudio de la organización (pp. 103-128). Colombia: Editorial Universidad Nacional de Colombia.

Londoño-Cardozo, J. (2018). Educación para emprender ¿práctica o teoría? Revista Argentina de Investigación en Negocios, 4(1), 97-106. Recuperado de http://ppct.caicyt.gov.ar/index.php/rain/issue/view/V4N1/showToc

López-Obando, P. (2017). Surgimiento de empresas catalogadas como spin-off universitarias en Colombia, análisis desde la gerencia de proyectos (fase I). Revista EAN, (82), 61-72. DOI: https://doi.org/10.21158/01208160.n82.2017.1649

Lowe, R. (2002). Invention, Innovation, and Entrepreneurship: The Commercialization of University Research by Inventor-founded Firms (Doctoral dissertation in Management). University of California, Berkeley.

Marulanda-Valencia, F., Montoya-Restrepo, I. \& Vélez-Restrepo, J. (2014). Aportes teóricos y empíricos al estudio del emprendedor. Cuadernos de Administración, 30(51), 89-99. Recuperado de http://www.redalyc.org/articulo.oa

Méndez-Méndez, M., Rebollar-Hernández, J., Lino-Gamiño, J., \& González-Pérez, M. (2015). Spin Off: Una Revisión Literaria en la Creación de Empresas Universitarias. Revista de Estrategias del desarrollo empresarial, 1(1), 1-14. Recuperado de http://www.ecorfan.org/spain/rj_estrategias_de.php

Mercado Pago ya está disponible para comercios de Colombia. (16 de julio de 2016). El tiempo. Recuperado de https://www.eltiempo.com/archivo/documento/CMS-16646959

Montoya-Pineda, D. (2016). Startup y Spinoff: definiciones, diferencias y potencialidades en el marco de la economía del comportamiento. Contexto, 5, 141-152. DOI: https://doi.org/10.18634/ctxj.5v.0i.657 
Mustar, P., Renault, M., Colombo, M., Piva, E., Fontes, M., Lockett, A. \& Moray, N. (2006). Conceptualising the heterogeneity of research-based spin-offs: A multi-dimensional taxonomy. Research Policy, 35(2), 289-308. DOI: https://doi.org/10.1016/j.respol.2005.11.001

Naranjo-Africano, G. (2011). Spin-off académica en Colombia: estrategias para su desarrollo. Multiciencias, 11(1), 35-43. Recuperado de http://www.redalyc.org/articulo.oa

Narváez-Vásquez, G., Maridueña-Arroyave, M., Chávez-Ferreiro, J. \& González-Garcilazo, M. (2016). Las spin off universitarias: revisión de la literatura sobre la ambigüedad del constructo. Revista Global de Negocios, 4(7), 95-108. Recuperado de https://www.theibfr.com/wpfb-file/rgn-v4n7-2016-9pdf/

Nicolaou, N. \& Birley, S. (2003a). Academic networks in a trichotomous categorisationof university spinouts. Journal of Business Venturing, 18(3), 333-359. DOI: https://doi.org/10.1016/S08839026(02)00118-0

Nicolaou, N., \& Birley, S. (2003b). Social Networks in Organizational Emergence: The University Spinout Phenomenon. Management Science, 49(12), 1702-1725. DOI: https://doi.org/10.1287/mnsc.49.12.1702.25116

Nlemvo, F., Pirnay, F. \& Surlemont, B. (2002). A stage model of academic spin-off creation. Technovation, 22(5), 281-289. DOI: https://doi.org/10.1016/S0166-4972(01)00019-0

OECD. (2002). Special Issue on New Science and Technology Indicators, STI Review, 2000(2), 97-120. DOI: https://doi.org/10.1787/sti_rev-v2000-2-en

Ospina-Díaz, L. Cifuentes-Leiton, D., Gaitán-Vera, B. \& Londoño-Cardozo, J. (2019). La RSO y la Economía Colaborativa: principios de análisis. Revista Argentina de Investigación en Negocios, 5(1), 73-80. Recuperado de http://ppct.caicyt.gov.ar/index.php/rain/issue/view/v5n1/showToc

Pineda-Márquez, K., Morales-Rubiano, M. \& Ortíz-Riaga, C. (2011). Modelos y mecanismos de interacción universidad-empresa-Estado: retos para las universidades colombianas. Equidad \& Desarrollo, (15), 41-67. Recuperado de https://dialnet.unirioja.es/servlet/articulo?codigo=5166538

Pinzón-Escobar, C. (2016). Puentes entre la teoría y la práctica: la experimentación de las medidas descriptivas con el laboratorio de empresa. En Beltrán-Duque, A., Echeverry-Romero, R., Restrepo-Rivillas \& Navarro-hernández, A. La investigación en administración y la responsabilidad de la academia en mercados globalizados (pp. 970-990). Santa Marta: Fundación Universidad Externado de Colombia. 
Pinzón-Escobar, C. \& Pineda-Fajardo, F. (2017). El laboratorio de empresa, modelo didáctico para la consolidación de los conocimientos de la mezcla de mercadeo. Trabajo presentado en el Congreso Reflexiones sobre Administración. Tendencias actuales de investigación Administrativa, Palmira. Recuperado de http://bdigital.unal.edu.co/71083/

Pinzón-Escobar, C. (2019). Laboratorio de organización: modelo didáctico para el proceso de enseñanza aprendizaje de los estudiantes de administración de empresas. En Beltrán-Duque, A., EcheverryRomero, R., Restrepo-Rivillas, C. \& Rodríguez-Orejuela, A. Las nuevas tecnologías y los desafíos para la administración (pp. 3262-3281). Bogotá: Fundación Universidad Externado de Colombia.

Pirnay, F. (2001). La valorisation economique des resultats de recherche universitaire par creation d'activites nouvelles (spin-offs universitaires): proposition d'un cadre procedutal d'essaimage. (Tesis Doctoral, Université Toulouse).

Rappert, B., Webster, A. \& Charles, D. (1999). Making sense of diversity and reluctance: academicindustrial relations and intellectual property. Research Policy, 28(8), 873-890. DOI: https://doi.org/10.1016/S0048-7333(99)00028-1

Real Academia Española-RAE. (2014). Diccionario de la lengua española. Edición del Tricentenario. Recuperado de https://dle.rae.es/?id=GEBq7jT

Ríos-Szalay, J. (2010). La estandarización terminológica de la administración, un empeño amplio y persistente, ¿pero inviable? Congreso Internacional de Contaduría, Administración e Informática. Memorias. México: Universidad Nacional Autónoma de México.

Rodeiro-Pazos, D., Calvo-Babío, N., \& Fernández-López, S. (2012). La gestión empresarial como factor clave de desarrollo de las spin-offs universitarias. Análisis organizativo y financiero. Cuadernos de Gestión, 12(1), 59-81. Recuperado de https://www.redalyc.org/articulo.oa

Shane, S. (2004). Academic Entrepreneurship: University Spinoffs and Wealth Creation. $1^{\text {a }}$ ed. United Kingdon: Edward Elgar Publishing.

Smilor, R., Gibson, D. \& Dietrich, G. (1990). University spin-out companies: Technology start-ups from UT-Austin. Journal of Business Venturing, 5(1), 63-76. DOI. https://doi.org/10.1016/08839026(90)90027-Q

Stankiewicz, R. (1994). Spin-off companies from universities. Science and Public Policy, 21(2), 99-107. DOI: https://doi.org/10.1093/spp/21.2.99 
Steffensen, M., Rogers, E. \& Speakman, K. (2000). Spin-offs from research centers at a research university. Journal of Business Venturing, 15(1), 93-111. DOI: https://doi.org/10.1016/S08839026(98)00006-8

Tang, K. Vohora, A. \& Freeman, R. (2004). Taking Research to Market: How to Build and Invest in Successful University Spinouts. London: Euromoney Institutional Investor.

Tello-Castrillón, C. (2018). El concepto de organización, tan cerca y tan lejos. En Tello-Castrillón, C., \& Pineda-Henao, E. (Comp.) Conjeturas organizacionales: fundamentos para el estudio de la organización (pp. 79-102). Bogotá: Editorial Universidad Nacional de Colombia.

Tübke, A. (2004). Success Factors of Corporate Spin-Offs. USA: Springer US.

Van Dierdonck, R. \& Debackere, k. (1988). Academic entrepreneurship at Belgian Universities. $R \& D$ Management, 18(4), 341-353. DOI: https://doi.org/10.1111/j.1467-9310.1988.tb00609.x

Zuluaga-Esquivel, M. (2017). Modelo SAS, figura jurídica preferida para crear empresa. Revista Universidad EAFIT, 52(169), 50-53. Recuperado de http://publicaciones.eafit.edu.co/index.php/revista-universidad-eafit/article/view/5666

Zúñiga-González, C. (2011). Texto básico de Economía Agrícola: Su importancia para el desarrollo local sostenible. $1^{\text {a }}$ ed. Nicaragua: Editorial universitaria, UNAN-LEON. 